\title{
A General Method for Calculating Elastic Moduli of Helical Polymer Chains in Crystals; Application to Poly(Oxymethylene)
}

\author{
Hiromu Sugeta and Tatsuo Miyazawa \\ Institute for Protein Research, Osaka University Joancho, Kitaku, Osaka, Japan.
}

(Received November 8, 1969)

\begin{abstract}
A new general method was derived for calculating elastic moduli of isolated polymer-chains and of polymer-chains in crystals. Equations for elastic moduli were written with the $B$ submatrices as used in normal coordinate treatments. The new method was applied for calculating the elastic moduli of (poly)oxymethylene chains in trigonal and orthorhombic crystals.
\end{abstract}

KEY WORDS Polymer / Young's Modulus / Elastic Modulus / Intrachain Potential / Helical Structure / Poly(Oxymethylene) /

Force fields of chain-polymer crystals are much more anisotropic than those molecular of crystals, and the intrachain restoring force along the chain direction is much stronger than interchain restoring forces. Because of this strong anisotropy of force fields in chain-polymer crystals, the elastic modulus in the direction of the chain axis may be reasonably treated with the single-chain approximation.

Elastic moduli of polymer chains were previously calculated by Meyer and Lotmar, ${ }^{1}$ Lyons $^{2}$, and Treloar $^{3}$, with the use of bond-stretching and bond-angle bending force constants. However, general methods for calculating elastic moduli of helical polymer chains were derived by Shimanouchi et $a .^{4}$ and by Miyazawa ${ }^{5}$.

The repeating regularity of a helical polymerchain is characterized with the unit translation (d) along the helix axis and the unit twist $(\theta)$ about the axis (Figure 1), ${ }^{6-8}$ while the intrachain potential is usually expressed with bond-stretching $(\Delta r)$, angle-bending $(\Delta \phi)$ and internal-rotation coordinates $(\Delta \tau)$. Accordingly, Shimanouchi, Asahina and Enomoto ${ }^{4}$ derived the equations for elastic moduli from the relation ${ }^{6,7}$ between the unit translation $(d)$ and intramolecular parameters $(r, \phi$, and $\tau)$. On the other hand, Miyazawa ${ }^{5}$ derived the equations for elastic moduli and elastic strains from the relation between intramolecular parameters and helical parameters $\left(\rho_{i}, d_{i j}\right.$, and $\left.\theta_{i j}\right) .^{7}$ In these previous studies, elastic moduli of isolated polymer-chains were calculated without the restriction of $\Delta \theta=0$.

However, if a polymer crystal is subject to a homogeneous deformation, translational symmetries of the three-dimensional crystal lattice are reserved and constituent polymer-chains are deformed so that the unit translation is changed $(\Delta d \neq 0)$ but the unit twist is kept constant $(\Delta \theta=0)$. Accordingly, for calculating elastic moduli of polymer-chains in crystals, previous methods $^{4,5}$ can not be applied.

In the present study, therefore, a new general method is derived for calculating elastic moduli of isolated polymer-chains $(\Delta \theta \neq 0)$ and of polymer-chains in crystals $(\Delta \theta=0)$. Equations for elastic moduli are written with $B$ submatrices ${ }^{9}$ as used for normal-vibration treatments of helical polymer chains.

\section{HOMOGENEOUS DEFORMATION OF POLYMER CHAINS}

Elastic deformation of an isolated polymerchain is assumed to be uniform under a tension along the chain axis. A homogeneous deformation of a helical chain may be described with changes in unit translation $(\Delta d)$ and in unit twist $(\Delta \theta)$. Accordingly, the external-strain vector $\widetilde{U}=\left[\begin{array}{ll}u_{1} & u_{2}\end{array}\right]$ may be defined as

$$
\begin{aligned}
& u_{1}=u_{d}=\Delta d / d \\
& u_{2}=u_{\theta}=\Delta \theta
\end{aligned}
$$

where a tilde denotes the transpose of a matrix. 


\section{Elastic Moduli of Polymer Chains}

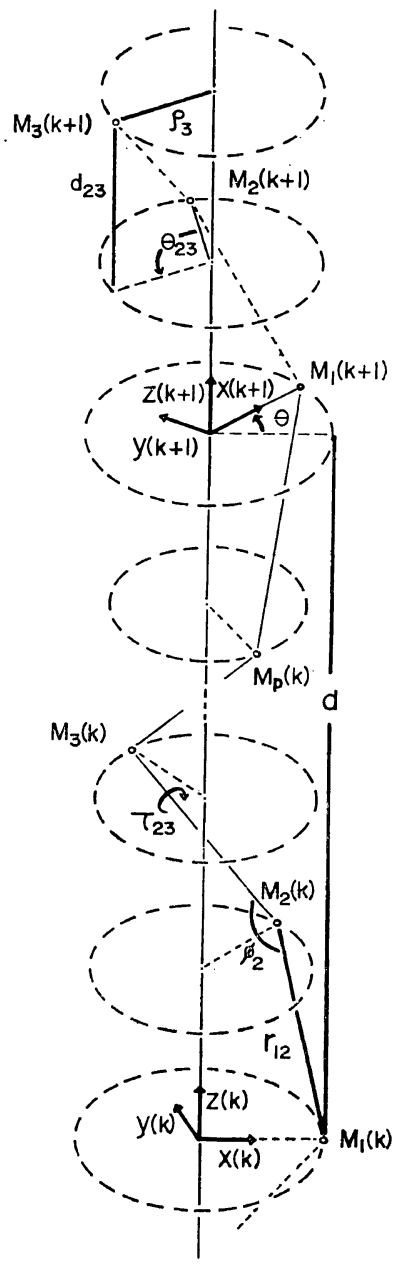

Figure 1. Helix parameters and intramolecular parameters.

For treating elastic moduli of isolated polymerchains, $u_{1}$ and $u_{2}$ are both used as external strains. However, for treating elastic moduli of polymer chains in crystals, only $u_{1}$ is used as the external strain and rows and columns associated with $u_{2}$ should be dropped in succeeding equations.

\section{ELASTIC MODULI OF HELICAL POLYMER CHAINS}

For describing displacements of chain atoms under a homogeneous deformation, right-handed Cartesian coordinates $\tilde{x}(k)=[x(k) y(k) z(k)]$ of the $k$-th repeat unit are defined as shown in
Figure 1; the $z(k)$ axis rests on the helix axis. The Cartesian displacement vector $\boldsymbol{X}(k)$ of the $k$-th unit with $p$ atoms $\left[\mathbf{M}_{1}(k) \mathbf{M}_{2}(k) \ldots \mathbf{M}_{p}(k)\right]$ are given as,

$$
\begin{array}{r}
\tilde{\boldsymbol{X}}(k)=\left[\Delta x_{1}(k) \Delta y_{1}(k) \Delta z_{1}(k) \Delta x_{2}(k) \Delta y_{2}(k) \Delta z_{2}(k)\right. \\
\left.\ldots \Delta x_{p}(k) \Delta y_{p}(k) \Delta z_{p}(k)\right]
\end{array}
$$

The displacement vector $\boldsymbol{X}(k)$ of the $k$-th unit is transformed into the vector $\boldsymbol{X}(k+1)$ of the $(k+1)$-th unit by the screw operation with the unit translation $(d)$ and the unit twist $(\theta)$.

For a homogeneous deformation of an isolated chain, atomic displacements are related with external strains,

$$
\begin{aligned}
& \Delta x_{i}(k+m)-\Delta x_{i}(k)=-y_{i} m \Delta \theta=-m y_{i} u_{2} \\
& \Delta y_{i}(k+m)-\Delta y_{i}(k)=x_{i} m \Delta \theta=m x_{i} u_{2} \\
& \Delta z_{i}(k+m)-\Delta z_{i}(k)=m \Delta d=m d u_{1}
\end{aligned}
$$

where $x_{i}$ and $y_{i}$ are coordinates of the $i$-th atom. Eq. 3 may be rewritten in matrix forms,

$$
\boldsymbol{X}(k+\boldsymbol{m})-\boldsymbol{X}(k)=\boldsymbol{m} \boldsymbol{T} \boldsymbol{U}
$$

where $\boldsymbol{T}$ is given as

$$
\begin{aligned}
& \widetilde{\boldsymbol{T}}=\left[\begin{array}{lll}
\tilde{t}_{1} & \tilde{\boldsymbol{t}}_{2} & \ldots \\
\tilde{\boldsymbol{t}}_{p}
\end{array}\right] \\
& \boldsymbol{t}_{i}=\left[\begin{array}{cc}
0 & -y_{i} \\
0 & x_{i} \\
d & 0
\end{array}\right]
\end{aligned}
$$

External strains $\left(u_{1}\right.$ and $\left.u_{2}\right)$ of a homogeneous deformation are accompanied with atomic displacements of each unit so that the potential energy is minimized or, in other words, the internal stresses vanish. Defining internal strains $(\xi)$ as $\boldsymbol{X}(0)$, the atomic displacements $\boldsymbol{X}(m)$ of the $m$-th repeat unit may be expressed as

$$
\boldsymbol{X}(m)=\boldsymbol{\xi}+\boldsymbol{m} \boldsymbol{T} \boldsymbol{U}
$$

The potential energy (harmonic terms) of a polymer chain per unit volume may be expressed in terms of internal coordinates,

$$
2 V=(1 / N A d) \sum_{k, m} \tilde{\boldsymbol{R}}(k+m) \boldsymbol{F}(k) \boldsymbol{R}(m)
$$

where $N$ is the number of repeat units, $A$ is the cross section per molecular chain, $\boldsymbol{R}(m)$ is the internal coordinate vector of the $m$-th repeat unit, and $\boldsymbol{F}(k)$ is the potential energy submatrix associated with $\tilde{\boldsymbol{R}}(k+m)$ and $\boldsymbol{R}(m)$, and 


\section{H. Sugeta and T. Miyazawa}

$$
\boldsymbol{F}(-k)=\tilde{\boldsymbol{F}}(k)
$$

For a homogeneous deformation, internal coordinates of all the units $(m)$ are common $\boldsymbol{R}(m)=\boldsymbol{R}$. Accordingly, the potential energy per unit volume may be rewritten as

$$
2 V=(1 / A d) \sum_{k} \tilde{\boldsymbol{R}} \boldsymbol{F}(k) \boldsymbol{R}=(1 / A d) \tilde{\boldsymbol{R}}_{0} \boldsymbol{R}
$$

where

$$
\boldsymbol{F}_{0}=\sum_{k} \boldsymbol{F}(k)
$$

For a helical polymer chain, the internal coordinates $\boldsymbol{R}(k)$ and Cartesian displacement coordinates $\boldsymbol{X}(k)$ of the $k$-th repeat unit are transformed into $\boldsymbol{R}(k+1)$ and $\boldsymbol{X}(k+1)$, respectively, of the $(k+1)$-th unit by the operation of screwrotation. Accordingly the $\boldsymbol{B}$ matrix for a helical polymer is expressed in terms of the $\boldsymbol{B}(m)$ submatrices, ${ }^{9}$

$$
\begin{aligned}
(2 A d) V= & \tilde{\boldsymbol{\xi}} \tilde{\boldsymbol{B}}_{0} \boldsymbol{F}_{0} \boldsymbol{B}_{0} \xi+\tilde{\boldsymbol{\xi}} \tilde{\boldsymbol{B}}_{0} \boldsymbol{F}_{0} \boldsymbol{B}_{u} \boldsymbol{U} \\
& +\tilde{\boldsymbol{U}} \tilde{\boldsymbol{B}}_{u} \boldsymbol{F}_{0} \boldsymbol{B}_{0} \boldsymbol{\xi}+\tilde{\boldsymbol{U}} \tilde{\boldsymbol{B}}_{u} \boldsymbol{F}_{0} \boldsymbol{B}_{u} \boldsymbol{U} \\
= & \tilde{\boldsymbol{\xi}} \boldsymbol{F}_{\xi} \xi+2 \tilde{\xi} \boldsymbol{F}_{\xi u} \boldsymbol{U}+\tilde{\boldsymbol{U}} \boldsymbol{F}_{u} \boldsymbol{U}
\end{aligned}
$$

where

$$
\begin{aligned}
\boldsymbol{F}_{\xi} & =\tilde{\boldsymbol{B}}_{0} \boldsymbol{F}_{0} \boldsymbol{B}_{0} \\
\boldsymbol{F}_{u} & =\tilde{\boldsymbol{B}}_{u} \boldsymbol{F}_{0} \boldsymbol{B}_{u} \\
\boldsymbol{F}_{\xi u} & =\tilde{\boldsymbol{B}}_{0} \boldsymbol{F}_{0} \boldsymbol{B}_{u}
\end{aligned}
$$

\begin{tabular}{|c|c|c|c|c|}
\hline & $\ldots \tilde{\boldsymbol{X}}(\mathrm{k}-m) \ldots \tilde{\boldsymbol{X}}(k-1)$ & $\tilde{\boldsymbol{X}}(k)$ & $\tilde{\boldsymbol{X}}(k+m) \ldots$ & \\
\hline . & $\ldots \cdot \cdot$ & . & $\begin{array}{cccc}\cdot & \ldots & \cdot & \ldots\end{array}$ & \\
\hline $\boldsymbol{R}(k-1)$ & $\ldots \boldsymbol{B}(m-1) \ldots \boldsymbol{B}(0)$ & $B(-1)$ & $B(-2) \ldots B(-m-1) \ldots$ & \\
\hline $\boldsymbol{R}(k)$ & $\ldots \boldsymbol{B}(m) \quad \ldots B(1)$ & $B(0)$ & $\boldsymbol{B}(-1) \ldots \boldsymbol{B}(-m) \quad \ldots$ & \\
\hline $\boldsymbol{R}(k+1)$ & $\ldots B(m+1) \ldots B(2)$ & $B(1)$ & $\boldsymbol{B}(0) \quad \ldots B(-m+1) \ldots$ & \\
\hline$\cdot$ & $\ldots \quad \cdot \quad \ldots \quad \cdot$ & $\cdot$ & $\ldots \quad \cdot \quad \cdots$ & (12) \\
\hline
\end{tabular}

Under a given external strain $(\boldsymbol{U})$, the internal strains $(\xi)$ are derived from the condition of minimizing potential energy $(V)$ or from the condition of null internal stresses $\left(\partial V / \partial \xi_{i}=0\right)$.

$$
\begin{aligned}
& \boldsymbol{F}_{\xi \xi}+\boldsymbol{F}_{\xi u} \boldsymbol{U}=0 \\
& \boldsymbol{\xi}=-\boldsymbol{F}_{\xi}^{-1} \boldsymbol{F}_{\xi u} \boldsymbol{U}
\end{aligned}
$$

The singularity of the $\boldsymbol{F}_{\xi}$ matrix will be discussed
In general, the internal coordinates $\boldsymbol{R}(k)$ are related to the Cartesian displacement coordinates $\boldsymbol{X}(m)$ as,

$$
\boldsymbol{R}(k)=\sum_{m} \boldsymbol{B}(m) \boldsymbol{X}(k-m)
$$

From Eqs. 7 and 13, uniform internal displace-

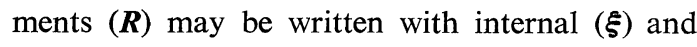
external strains $(U)$,

$$
\begin{aligned}
\boldsymbol{R} & =\sum_{m} \boldsymbol{B}(m) \boldsymbol{X}(-m)=\sum_{m} \boldsymbol{B}(m)[\boldsymbol{\xi}-m \boldsymbol{T} \boldsymbol{U}] \\
& =\boldsymbol{B}_{0} \boldsymbol{\xi}+\boldsymbol{B}_{u} \boldsymbol{U}
\end{aligned}
$$

where

$$
\begin{aligned}
\boldsymbol{B}_{0} & =\sum_{m} \boldsymbol{B}(m) \\
\boldsymbol{B}_{u} & =-\left[\sum_{m} m \boldsymbol{B}(m)\right] \boldsymbol{T}
\end{aligned}
$$

Substituting Eq. 14 into Eq. 10, the potential energy $(V)$ is given as later. The uniform deformations $(\boldsymbol{R})$ of internal coordinates are then derived from Eqs. 14 and 22.

$$
\boldsymbol{R}=\left(\boldsymbol{B}_{u}-\boldsymbol{B}_{0} \boldsymbol{F}_{\xi}^{-1} \boldsymbol{F}_{\xi u}\right) \boldsymbol{U}
$$

The potential energy $(V)$ is now expressed in terms of external strains $(U)$.

$$
2 V=(1 / A d) \tilde{U}\left(\boldsymbol{F}_{u}-\tilde{\boldsymbol{F}}_{\xi u} \boldsymbol{F}_{\xi}^{-1} \boldsymbol{F}_{\xi u}\right) \boldsymbol{U}=\tilde{\boldsymbol{U}} \boldsymbol{C} \boldsymbol{U}
$$

where $C$ is the elastic constant matrix as given by

$$
\boldsymbol{C}=(1 / A d)\left(\boldsymbol{F}_{u}-\tilde{\boldsymbol{F}}_{\xi u} \boldsymbol{F}_{\xi}^{-1} \boldsymbol{F}_{\xi u}\right)
$$

External stress parameters, $f_{i}\left(=\partial V / \partial u_{i}\right)$, are related to the external strain parameters as,

$$
\boldsymbol{f}=\boldsymbol{C} \boldsymbol{U}
$$

or alternately

$$
\boldsymbol{U}=\boldsymbol{C}^{-1} \boldsymbol{f}=\boldsymbol{S} \boldsymbol{f}
$$

where $S\left(=C^{-1}\right)$ is the compliance matrix.

From Eqs. 22, 23, and 27, the internal strains 


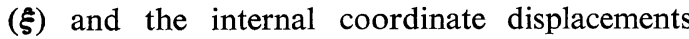
$(\boldsymbol{R})$ under given external stresses $(\boldsymbol{f})$ are given as,

$$
\begin{aligned}
& \boldsymbol{\xi}=-\boldsymbol{F}_{\xi}^{-1} \boldsymbol{F}_{\xi u} \boldsymbol{S} \boldsymbol{f} \\
& \boldsymbol{R}=\left(\boldsymbol{B}_{u}-\boldsymbol{B}_{0} \boldsymbol{F}_{\xi}^{-1} \boldsymbol{F}_{\xi u}\right) \boldsymbol{S} \boldsymbol{f}
\end{aligned}
$$

\section{Isolated Polymer Chains}

The elastic modulus $(E)$ along the chain axis is defined as,

$$
E=\frac{1}{\left(\partial u_{d} / \partial f_{d}\right)} .
$$

Under a homogeneous deformation of an isolated polymer chain $\left(u_{2} \neq 0\right)$,

$$
\partial u_{d} / \partial f_{d}=S_{11}
$$

and, accordingly the elastic modulus is given as,

$$
E=\frac{1}{S_{11}}
$$

\section{Polymer Chains in Crystal}

Under a homogeneous deformation of a polymer crystal, the unit translation is changed $\left(u_{1} \neq 0\right)$ but the unit twist is kept constant $\left(u_{2}=0\right)$. Then,

$$
f_{d}=C_{11} u_{d}
$$

and accordingly the elastic modulus of helical polymer chains in crystal is given as

$$
E=C_{11}
$$

\section{SINGULARITY OF $\boldsymbol{F}_{\xi}$ MATRIX}

The $\boldsymbol{F}_{\xi}$ matrix of Eq. 18 is the potential energy matrix for infinitesimal atomic displacements totally symmetric with respect to the operations of screw-rotations. Among totally symmetric displacements of chain atoms, there are two overall displacements, namely the translation along the chain axis and the rotation about the chain axis. Accordingly, the $\boldsymbol{F}_{\xi}$ matrix has two null eigenvalues corresponding to the overall translation and rotation. Since the $\boldsymbol{F}_{\xi}$ matrix is singular, the $\boldsymbol{F}_{\xi}^{-1}$ matrix will be defined as follows:

The $\boldsymbol{F}_{\xi}$ matrix is diagonalized by the orthogonal transformation.

$$
\begin{aligned}
\xi & =L \eta \\
\tilde{L} F_{\xi} L & =\Lambda
\end{aligned}
$$

where $\boldsymbol{L}$ is the eigenvector matrix $\left(\tilde{\boldsymbol{L}}=\boldsymbol{L}^{-1}\right)$ and
$\boldsymbol{A}$ is the eigenvalue matrix with two null diagonal elements. Since the two null eigenvalues are due to the overall translation and rotation, the $\eta$ coordinate vector is partitioned into two subvectors, $\eta^{*}$ and $\eta^{\circ}$, which are the $\eta$ coordinates for the $3 p-2$ genuine internal motions and for the overall translation and rotation, respectively. Accordingly, the eigenvector matrix $\boldsymbol{L}$ is also partitioned into $L^{*}[3 p \times(3 p-2)]$ and $L^{\circ}$ $(3 p \times 2)$, and the eigenvalue matrix $\Lambda$ is factored into the $\Lambda^{*}$ matrix with $3 p-2$ nonzero diagonal elements and a zero matrix (0).

$$
\eta=\left[\begin{array}{l}
\eta^{*} \\
\eta^{\circ}
\end{array}\right] \quad L=\left[L^{*} L^{\circ}\right] \quad \Lambda=\left[\begin{array}{cc}
\Lambda^{*} & 0 \\
0 & 0
\end{array}\right]
$$

For the new matrices $L^{*}$ and $\Lambda^{*}$, the following relations are derived:

$$
\begin{aligned}
& \tilde{\boldsymbol{L}}^{*} \boldsymbol{F}_{\xi} \boldsymbol{L}^{*}=\boldsymbol{\Lambda}^{*} \\
& \boldsymbol{L}^{*} \boldsymbol{\Lambda}^{*} \tilde{\boldsymbol{L}}^{*}=\boldsymbol{F}_{\xi} \\
& \tilde{\boldsymbol{L}}^{*} \boldsymbol{L}^{*}=\boldsymbol{E}[(3 p-2) \times(3 p-2) \text { unit matrix }] \\
& \tilde{\boldsymbol{L}}^{\circ} \boldsymbol{F}_{\xi} \boldsymbol{L}^{\circ}=\mathbf{0} \\
& \tilde{\boldsymbol{L}}^{\circ} \boldsymbol{F}_{\xi} \boldsymbol{L}^{*}=\mathbf{0}
\end{aligned}
$$

The second term $\left(\boldsymbol{L}^{\circ} \boldsymbol{\eta}^{\circ}\right)$ on the right side in Eq. 36 is due to the overall translation and rotation, and is redundant in treating the potential energy associated with elastic deformations,

$$
\xi=L^{*} \eta^{*}
$$

Eq. 42 is now substituted into Eq. 21, yielding

$$
\boldsymbol{F}_{\xi} L^{*} \eta^{*}+\boldsymbol{F}_{\xi u} U=\mathbf{0}
$$

Multiplication by $\tilde{L}^{*}$ from the left side gives

$$
\begin{aligned}
& \boldsymbol{\Lambda}^{*} \boldsymbol{\eta}^{*}+\tilde{\boldsymbol{L}}^{*} \boldsymbol{F}_{\xi u} \boldsymbol{U}=\mathbf{0} \\
& \boldsymbol{\eta}^{*}=-\left(\boldsymbol{\Lambda}^{*}\right)^{-1} \tilde{\boldsymbol{L}}^{*} \boldsymbol{F}_{\xi u} \boldsymbol{U}
\end{aligned}
$$

From Eqs. 42 and 44,

$$
\boldsymbol{\xi}=-\boldsymbol{L}^{*}\left(\boldsymbol{\Lambda}^{*}\right)^{-1} \tilde{\boldsymbol{L}}^{*} \boldsymbol{F}_{\xi u} \boldsymbol{U}
$$

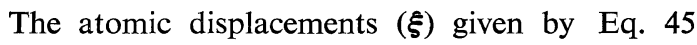
are independent of the overall translation and rotation (Eqs. 44 and 45 may also be derived from the property of the $B$ matrix $B_{0} L^{\circ}=0$ ). Comparing Eq. 45 with Eq. 22, the $\boldsymbol{F}_{\xi}^{-1}$ matrix may now be defined as

$$
\boldsymbol{F}_{\xi}^{-1}=\boldsymbol{L}^{*}\left(\boldsymbol{\Lambda}^{*}\right)^{-1} \tilde{\boldsymbol{L}}^{*}
$$




\section{H. Sugeta and T. Miyazawa}

The matrix products $\boldsymbol{F}_{\xi}^{-1} \boldsymbol{F}_{\xi}$ and $\boldsymbol{F}_{\xi} \boldsymbol{F}_{\xi}^{-1}$ are not unit matrices. However, the following equations are derived from Eqs. 37-39 and 46:

$$
\begin{aligned}
& \boldsymbol{F}_{\xi} \boldsymbol{F}_{\xi}^{-1} \boldsymbol{F}_{\xi}=\boldsymbol{F}_{\xi} \\
& \boldsymbol{F}_{\xi}^{-1} \boldsymbol{F}_{\xi} \boldsymbol{F}_{\xi}^{-1}=\boldsymbol{F}_{\xi}^{-1}
\end{aligned}
$$

\section{ELASTIC MODULUS OF POLY(OXYMETHYLENE)}

Poly(oxymethylene) (POM) has been found to exist in two crystalline forms, namely trigonal and orthorhombic. The molecular chain in the trigonal POM crystal takes a $9 / 5$ helix with nine repeat units $\left(\mathrm{CH}_{2} \mathrm{O}\right)$ and five turns per fiber period, while the molecular chain in the orthorhombic POM crystal takes a $2 / 1$ helix with two units and one turn per fiber period. For the trigonal POM crystal, the elastic modulus in the direction of the chain axis was measured as 0.053 mdyne $/ \AA^{2}$ by Sakurada et al. ${ }^{11}$

The elastic moduli of POM chains were calculated with the general method of the present study. The structure parameters used in calculations are bond lengths of $r(\mathrm{C}-\mathrm{O})=1.42 \AA$ and $r(\mathrm{C}-\mathrm{H})=1.09 \AA$, tetrahedral valence angles, and internal-rotation angles $\tau(\mathrm{C}-\mathrm{O})=76^{\circ} 8^{\prime}$ for the $9 / 5$ helix and $\tau(\mathrm{C}-\mathrm{O})=60^{\circ} 0^{\prime}$ for the $2 / 1$ helix. The unit translations are $d=1.866 \AA$ for the $9 / 5$ helix and $d=1.640 \AA$ for the $2 / 1$ helix. The cross sections per molecular chain are $A=17.31$ $\AA^{2}$ for the $9 / 5$ helix $^{12}$ and $A=18.25 \AA^{2}$ for $2 / 1$ helix ${ }^{13}$. The intrachain potential constants were refined previously, ${ }^{14}$ by the method of least squares, with reference to the observed vibrational frequencies of the trigonal POM crystal at room temperature. $^{15,16}$ Calculated results are summarized in Table I.

Under a homogeneous deformation of a polymer crystal, constituent polymer chains are deformed with the condition of $\Delta \theta=0$ and the elastic modulus of the $9 / 5$ helical chain in the trigonal crystal is calculated as $0.095 \mathrm{mdyne} / \AA^{2}$, as compared with the experimental value of 0.053 mdyne $/ \AA^{2}$. The elastic modulus of the $2 / 1$ helical chain in the orthorhombic crystal is calculated as $0.048 \mathrm{mdyne} / \AA^{2}$, although the experimental value is not available.

The $9 / 5$ helical chain of trigonal POM is calculated to be much stiffer than the $2 / 1$ helical
Table I. Changes in helix parameters $(\rho, d$ and $\theta)$ and intramolecular parameters $(r, \phi \text { and } \tau)^{\mathrm{a}}$ accompanying the chain-extension of POM through $1 \%$

\begin{tabular}{lcc}
\hline & $\begin{array}{c}\text { Trigonal } \\
(9 / 5 \text { helix })\end{array}$ & $\begin{array}{c}\text { Orthorhombic } \\
(2 / 1 \text { helix })\end{array}$ \\
\hline$\Delta \rho_{O}$ & -0.57 & -0.51 \\
$\Delta \rho_{C}$ & -0.32 & -0.27 \\
$\Delta d_{C O}$ & 0.93 & 0.82 \\
$\Delta \theta C o$ & 0 & 0 \\
\hline$\Delta r(\mathrm{C}-\mathrm{O})$ & 0.10 & 0.03 \\
$\Delta \phi(\mathrm{C}-\mathrm{O}-\mathrm{C})$ & 1.17 & 0.82 \\
$\Delta \phi(\mathrm{O}-\mathrm{C}-\mathrm{O})$ & 0.83 & 0.57 \\
$\Delta \tau(\mathrm{C}-\mathrm{O})$ & 0.89 & 0.84
\end{tabular}

a $\Delta \rho, \Delta d$, and $\Delta r$ are given in $\AA / 100$ and $\Delta \theta, \Delta \phi$, and $\Delta \tau$ are given in radian/100.

chain of orthorhombic POM; the force required to stretch a chain through $1 \%$ is calculated as 0.0170 mdyne for the $9 / 5$ helical chain which is twice as strong as the force $(0.0088$ mdyne) for the $2 / 1$ helical chain. This difference in stiffness is due to the difference in chain-conformation; the internal-rotation angle is $76^{\circ}$ for the $9 / 5$ helical chain and $60^{\circ}$ for the $2 / 1$ helical chain. Possibly, the elastic modulus in the direction of the chain axis is fairly sensitive to chain conformations of helical polymers.

Finally it may be remarked that the calculated elastic moduli of isolated POM chains $(\Delta \theta \neq 0)$ are much smaller than those of POM chains in crystals $(\Delta \theta=0)$; the calculated values of $E(\Delta \theta \neq 0)$ are as small as $0.040 \mathrm{mdyne} / \AA^{2}$ for the 9/5 helical chain and as $0.019 \mathrm{mdyne} / \AA^{2}$ for the $2 / 1$ helical chain. However, for trigonal POM crystal at room temperature, the X-ray study by Sakurada et al. ${ }^{11}$ indicates that the helix parameter $\theta$ does not change under stress and within the time scale of X-ray measurements. Accordingly, except for polymers with planar main chains, the elastic moduli of polymer chains previously calculated with the condition of $\Delta \theta \neq 0$ should be recalculated with the new condition of $\Delta \theta=0$.

\section{REFERENCES}

1. K. H. Meyer and W. Lotmar, Helv. Chim. Acta, 19, 68 (1936).

2. W. J. Lyons, J. Appl. Phys., 29, 1429 (1958); 30, 796 (1959).

3. L. R. G. Treloar, Polymer, 1, 95, 279, 290 (1960). 
4. T. Shimanouchi, M. Asahina, and S. Enomoto, J. Polym. Sci., 59, 93 (1962).

5. T. Miyazawa, Rept. Progr. Polym. Phys. Japan, 8, 47 (1965).

6. T. Shimanouchi and S. Mizushima, J. Chem. Phys., 23, 707 (1955).

7. T. Miyazawa, J. Polym. Sci., 55, 215 (1961).

8. H. Sugeta and T. Miyazawa, Biopolymers, 5, 673 (1967); 6, 1387 (1968).

9. H. Sugeta and T. Miyazawa, J. Chem. Phys., 47, 2034 (1967).

10. E. B. Wilson, Jr., J. Chem. Phys., 7, 1047 (1939); 9, 76 (1941).
11. I. Sakurada, T. Ito, and K. Nakamae, J. Polym. Sci., C15, 75 (1966).

12. T. Uchida and H. Tadokoro, J. Polym. Sci., Part A, 5, 63 (1967).

13. G. A. Carazzolo and M. Mammi, J. Polym. Sci., Part A, 1, 965 (1963).

14. H. Sugeta, Ph. D. Thesis, Osaka University. 1969.

15. H. Tadokoro, M. Kobayashi, Y. Kawaguchi, A. Kobayashi, and S. Murahashi, J. Chem. Phys., 38, 703 (1963).

16. H. Sugeta, T. Miyazawa, and T. Kajiura, $J$. Polym. Sci., Part B, 7, 251 (1969). 